\title{
Technological Use Behaviors, Internet Addiction and Personality among Italian University Students
}

\author{
Eugenia Treglia, Rosella Tomassoni \\ Department of Human, Social and Health Sciences, University of Cassino and Southern Lazio, Cassino, Italy \\ Email: eugenia.treglia@gmail.com
}

How to cite this paper: Treglia, E., \& Tomassoni, R. (2018). Technological Use Behaviors, Internet Addiction and Personality among Italian University Students. Psychology, 9, 472-484.

https://doi.org/10.4236/psych.2018.93029

Received: March 12, 2018

Accepted: March 27, 2018

Published: March 30, 2018

Copyright (c) 2018 by authors and Scientific Research Publishing Inc. This work is licensed under the Creative Commons Attribution International License (CC BY 4.0).

http://creativecommons.org/licenses/by/4.0/

\section{(c) (i) Open Access}

\begin{abstract}
Aims of this study are to evaluate the technological use behaviors among university students and the relationship between the type of use/abuse of internet and some personality characteristics. The sample consists of 435 Italian university students. The Multidimensional Personality Profile (MPP) test and the Questionnaire about the Internet use, abuse and addiction (UADI), have been administered online but in the experimenter's presence. The use of the Internet in our sample is mainly not a problematical one. Significant correlations were found between Self regulation and Dissociation $(r=-0.36)$ and between Machiavellism/cynism and Escape $(r=0.36)$, Dissociation $(r=0.33)$ and Experimentation $(r=0.34)$. The results of the correlations suggest that the more people are able to set purposes, monitor actions, organise and deal with matters with order and method (Self-Regulation), the less they will tend to have dissociative experiences connected to the use of Internet (Dissociation). A high level of cynicism correspond a high tendency to use Internet to escape from reality, a high tendency to dissociation and to aggressive/transgressive behaviours online. In conclusion it is necessary to consider the complex psychological dynamics in the relationship between the subject and the technological tool.
\end{abstract}

\section{Keywords}

Use of Internet, Risk of Internet Addiction, Personality

\section{Introduction}

Given the pervasiveness of information technology in contemporary society, it is not easy to outline a picture of the psychological characteristics of the Internet users/abusers. 
In Internet addiction it seems important to evaluate the abusing behaviour of the subjects according to the possible pre-existing psychological and psychosocial variables. For this reason, in this research we'll analyze some personality characteristics that could be associated with a dysfunctional use of the Internet. The estimate of the addiction disorder incidence substantially varies according to the considered country, the adopted methodology of survey (online or not) and according to the used instruments. Generally, online surveys have the advantage to collect a great amount of data in a relatively short time (Eppright et al., 1999). Nevertheless, an online administration can especially select those subjects that use the Internet frequently or the subjects who think to have some problems with the procedures of using it. Surveys not managed online by random selection of subjects are an important way to approach the study about the Internet Related Psychopathology (IRP).

Up to 2010, our records show that a single study about the spreading of this case among the population was published, having employed a random offline sampling method.

This study reported about a very low spreading estimate, varying from $0.3 \%$ to $0.7 \%$ (Aboujaoude et al., 2006). The global picture of the Internet addiction prevalence, presents a varied and complex scenario, featuring a wide range of rates, from $1 \%$ to $25 \%$ (Perrella \& Caviglia, 2014). In Italy people suffering from Internet addiction are about $6 \%$, considering serious and moderate levels of addiction (Poli \& Agrimi, 2012). A higher prevalence of the disorder is highlighted in the male population, as male subjects seem to be more engaged in virtual activities with a high "addiction gradient", such as role playing games, gambling and virtual sex (Morahan-Martin \& Schumacher, 2000). Furthermore, if the prevalence of PIU (Pathological Internet Users) is higher among male subjects, the percentage of people showing an intermediate level of seriousness (MIU: Maladaptive Internet Users) is higher among female people. Several studies reveal a negative relationship between age and IAD: the younger subjects should be more vulnerable to this pathology (Tonioni et al., 2011; Khazaal et al., 2008). It is consistent to assume that the internet addicted people spend on average many hours on the net, as suggested by several studies published to substantiate this intuition (Young, 1998; Widyanto \& McMurran, 2004; Leung, 2004). An Internet addicted person can be connected from 40 to 80 hours per week, with sessions that can last up to 20 hours (Young, 1998; Greenfield, 1999). A level of 39 hours per week not for school and/or working purposes is considered a possible cut off reporting a problematic usage (Young, 1998), but there are no unanimously accepted data about the variable usage time. Some authors propose to point out 3.89 hours per day as the average connection time for people affected by PIU and 3.27 in MIU users (Chou \& Hsiao, 2000; Durkee et al., 2012). With reference to specific aspects of personality, there is an association between IAD and opposition or aggressive behaviours (Yen et al., 2007; Ko et al., 2007). Even the Sensation seeking is an important risk factor (Tsai \& Lin, 2003; Kim \& Haridakis, 2009), in particular some of its under-size aspects such as the disinhibition or susceptibility to 
boredom (Velezmoro et al., 2010). Low self-esteem seems to be one of the most powerful factors of risk (Ko et al., 2007) and generally, a narcissistic frailty seems to have an explanatory crucial role in the understanding of the etiology of PIU. Even high levels of shyness are associated to a slight increase of the risk to develop an Internet addiction (Caplan, 2002; Chak \& Leung, 2004). Young people feeling alone, try to overcome this condition through social online interaction (McKenna \& Bargh, 2000). In this case, the Internet provides a compensatory function towards unsatisfactory relationships (Papacharissi \& Rubin, 2000). A recent Italian study reports a correlation between IAD and alexithymia (Fiumana et al., 2011) in accordance with the results from studies about other forms of dependence, related to substances or behaviours connected to the inability to express and recognize one's emotions (Caretti et al., 2005; Parker et al., 2005). It is important also the temperamental dimension of the effortful control, defined as the ability to inhibit a dominant response and/or plan, and detect errors (Rothbart \& Bates, 2006). Otherwise, considering the protective factors, an internal "locus of control" can reduce the risk to develop an Internet addiction (Chak \& Leung, 2004; Kim \& Haridakis, 2009). It is a very significant protective factor on the empirical level, and points out that young people that are able to take their active role in getting around the world, be it a real or a virtual one, can keep within a safe and healthy Internet usage, considered as an asset, not as an alternative reality (Wallace, 1999).

\section{Aims}

This research aims to investigate the relationship between young people and new technologies, and, in particular, to describe their behaviour in technology usage.

The specific goals of this research are:

1) Improving the understanding of the types of internet use among young people, surveying the average time spent online weekly and the different types of internet use;

2) Evaluate, among a sample of Italian students, the percentage of individuals with technological use behaviours that can be considered at risk of developing the Internet Addiction Disorder;

3) Studying the relationship between behaviours in technological use and personality.

\section{Method}

\subsection{Participants}

The sample was made by a total amount of 435 Italian individuals, 225 female students and 210 male students. $80 \%$ of them are between 18 and 20 years old, $14 \%$ are between 21 and $23,3 \%$ are between 24 and 29 , while a remaining $3 \%$ are older than $30.52 \%$ of the students have got a technical or vocational qualification, or a high school diploma with specialisation in teacher training; $30 \%$ have got a high school diploma in classical, scientific or linguistic subjects and the 
remaining $18 \%$ have attended other schools. $56 \%$ of them have never been employed. 90\% of the surveyed students are unmarried. Almost all students (98\%) entered the first year of the Bachelor's degree in humanistic studies.

\subsection{Measures}

The Multidimensional Personality Profile (MPP) (Caprara, Barbaranelli, De Carlo, \& Robusto, 2006) assesses five fundamental areas of personality: 1) Agency, 2) Social-emotional intelligence, 3) Self-regulation, 4) Coping ability, 5) Innovation, 6) Self-introduction. The test consists of 152 statements that need to be answered through individuals agreement on a five-level-scale. A specified time is not prescribed for the test, but it is important to answer all the questions in the questionnaire. The test was standardised on a sample of about 2500 individuals, $70 \%$ of whom were enrolled in competitive situations (selection, evaluation). Different regulations were provided according to the type and situation of the administration (competitive vs not-competitive). The psychometric characteristics of the test were proved by the analysis of the standardised sample (exploratory and confirmatory factor analysis, reliability analysis, descriptive analysis).

The second questionnaire used is UADI, Questionnaire about the Internet use, abuse and addiction (Del Miglio, Gamba, \& Cantelmi, 2001), an effective instrument to detect psychological and psychopathological variables connected to the use of the internet. In Italy it has been validated on a sample of 244 individuals aged between 13 and 57, that had been using the internet for 6 months at least and for not less than 6 hours per week. It is made of 75 items, with answer mode on a five scores Likert scale (from absolutely false to absolutely true), provided according the following 5 factors: 1) Compensatory Escape (CE); 2) Dissociation (DIS); 3) Impact on the life (IMP); 4) Experimentation (SPE); 5) Addiction (ADD).

Every possible score in the five scales of the UADI has been converted into the T scale. According to this new scale, subjects exceeding score 70, that means two standard deviations diverging from the average, are considered pathological. Otherwise, below score 30, the subject presents a psychological condition that is very far from the inquired characteristic. Scores between 31 and 69 , as it is easily comprehensible, classify the subjects as normal.

\subsection{Procedure}

The sample used for the survey was recruited at the University of Cassino and Southern Lazio. Students, belonging to the faculty of Social and Human Sciences, were invited to take a digital device during lessons on previously decided days, to be able to connect to the Internet. Before students undergo the psychological reagents, they were given a password to access the online platform of the University, where the tests had been loaded. The reagents, made accessible to everyone, were compiled directly online and in the experimenter's presence. This procedure, to be administered online but in presence, besides facilitating data 
collection and analysis, permitted us to test a random sample not suffering from the most frequent bias of a completely online survey (the implicit self-selection of the subjects, offering themselves as candidates for the survey). Before being submitted to the psychological reagents, students filled an online form about general personal data (age, gender, degree, family status, working life) and some specific information about their use of the internet (connection time and purpose of use of the Net). Afterwards, a personality reagent was administered, the Multidimensional Personality Profile (MPP) (Caprara, Barbaranelli, De Carlo, \& Robusto, 2006), to assess five fundamental areas of the personality (Agency, Social and Emotional intelligence, Self Regulation, Coping Ability, Innovation) and the Internet use, abuse and addiction test (UADI) by Del Miglio, Gamba, \& Cantelmi (2001), a tool able to point out psychological and psychopathological variables connected to the use of the internet. Then the scoring of the tests and the descriptive analysis of the personal data were carried out. A correlation analysis was conducted to evaluate the intensity of the association between psychological and psychopathological variables connected to the use of the internet and some personality characteristics of the users.

\section{Results}

The analysis of the answers given by the subjects about the amount of time spent online weekly, shows the following percentage distribution: $36 \%$ of our sample state to spend connected to the Net from 6 to 12 hours per week, $22 \%$ are connected from 13 to 19 hours weekly, 13\% from 20 to 26 hours, $10 \%$ from 27 to 33 hours weekly, $7 \%$ of people are connected from 34 to 40 hours per week, $9 \%$ of the people give no answers, and finally $3 \%$ of the them state to spend over 40 hours weekly connected to the Web (see Figure 1).

As for the prevailing purposes of Internet use by the surveyed subjects (they could choose more than one possible answer to this question), the following results were achieved: $73 \%$ of them state to use the Net for study/work reasons (consequently for information searching and sharing), 53\% use social networks, $44 \%$ use chat rooms, $3 \%$ uses it for online games, $42 \%$ listen to and download music from the Internet; $21 \%$ use the Net for online shopping, $1 \%$ visit websites

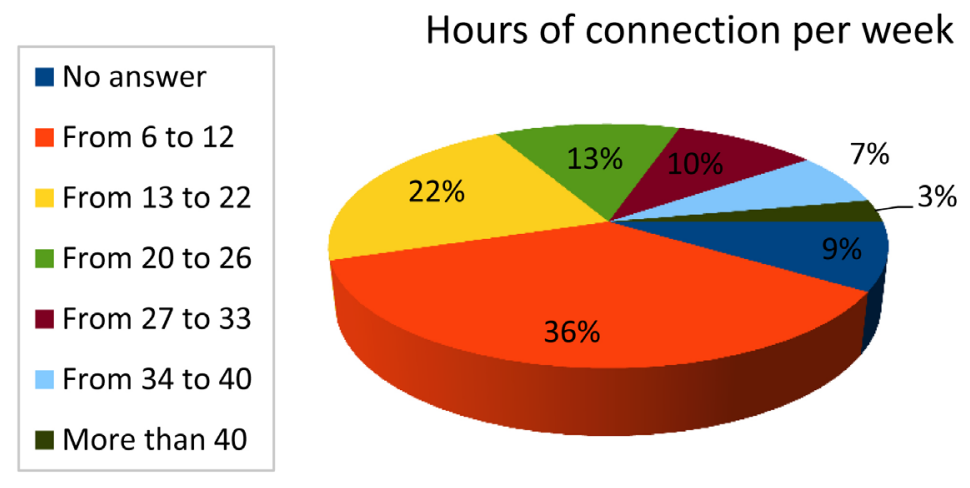

Figure 1. Hours of connection per week. 
for adults, and no individuals state to use the Net for online trading (see Figure 2).

\subsection{Test UADI Results}

From the exam of the data relating to the UADI questionnaire, it emerges that in the Compensatory Escape dimension 97\% of the individuals in our sample are on an average level (normal), while the remaining $3 \%$ are on a high level (pathological). The female subjects reach an average standardised score of 49 , while the males reach 45. In the Dissociation dimension, $98 \%$ of the individuals are on a medium level (normal), while the remaining $2 \%$ are on a high level (pathological). Females reach an average standardised score of 52, males reach 50 . In the Impact on the life dimension $93 \%$ of the subjects in the sample are on a medium level (normal), $6 \%$ on a low level (normal), $1 \%$ on a high level (pathological). Females reach an average standardized score of 66 (medium level), while males reach 73 (high level). In the Experimentation dimension 95\% of the individuals are on a medium level (normal), $3 \%$ on a low level (normal), and $2 \%$ on a high level (pathological). Females reach an average score of 54 (medium level), while males reach 52 (medium level). In the Addiction dimension 95\% of the surveyed individuals reach a medium level (normal), $4 \%$ reach a low level (normal), and $1 \%$ reach a high level (pathological). Both females and males reach an average standardised score of 51 (medium level).

\subsection{Test MPP Results}

Hereafter there are the results of the MPP Questionnaire analysis. In the Agency dimension female subjects in the sample get an average score of 44 (low), while male subjects get 52 (medium). In the Social/Emotional Intelligence dimension males get an average score of 53 (medium), and females get 49 (medium). In the

\section{Type of internet use}

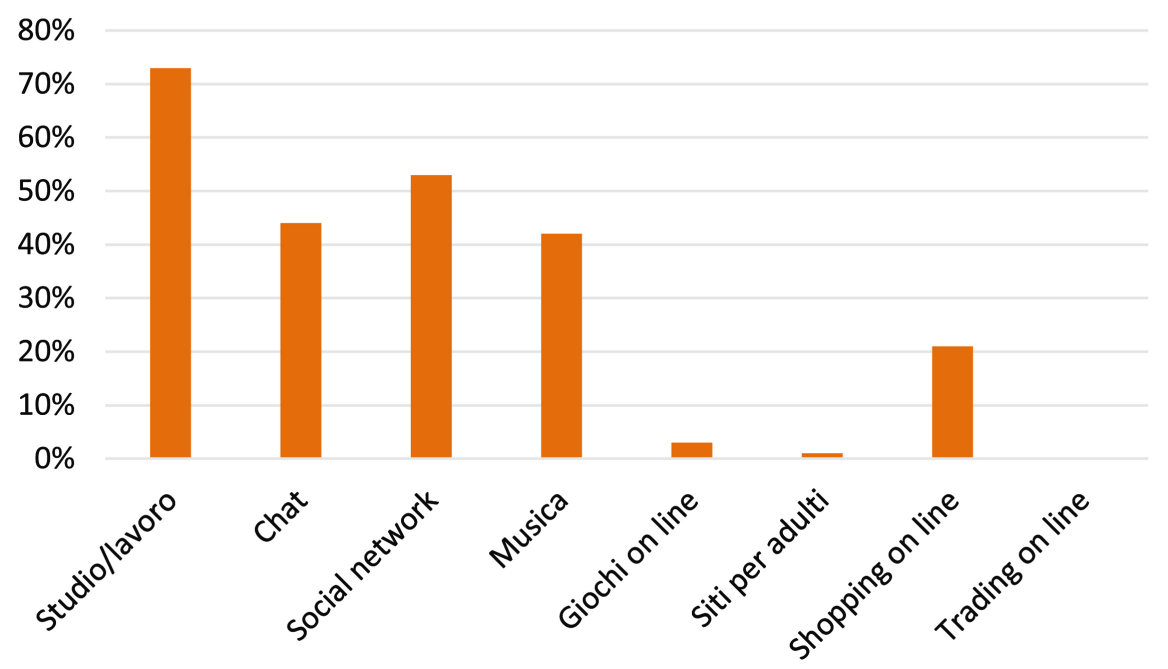

Figure 2. Type of internet use. 
Self-Regulation dimension males get the score of 46 (medium) and females get 51 (medium). In Coping ability males get an average score of 59 (high), while females get 48 (medium). In the Innovation dimension males get an average score of 54 (medium), and females get 50 (medium). As for Self-esteem males get an average score of 50 (medium), and females get 48 (medium). In the Social desirability dimension males get an average score of 54 (medium) and females get 49 (medium). Machiavellianism refers to the tendency of putting one's own interests before the ones of other people, and bending others and circumstances to one's interests and aims. In this dimension, males get an average score of 46 (medium), and females get 53 (medium). In the last evaluated characteristic, Impression management males get the average score of 46 (medium), and females of 51 (medium). The surveyed people show a normal interest in desiring to gain favour, appreciation and trust from other people.

The correlational analysis was conducted to examine the intensity of the association between the behaviour in technological use (UADI test scales) and the characteristics of personality (MPP test scales) and gave the correlation coefficients given in the Table 1 .

There is a weak negative correlation $(\mathrm{r}=-0.24)$ among the results got by the individuals in the Agency dimension and Dissociation dimension. It is also observed a weak negative correlation $(\mathrm{r}=-0.20)$ between the Social/Emotional intelligence and Experimentation dimensions. Self-regulation dimension is weakly negatively correlated to Escape ( $\mathrm{r}=-0.28)$ and to Experimentation $(\mathrm{r}=-0.26)$, while there is a significant negative correlation between Self regulation and Dissociation $(\mathrm{r}=-0.36)$. A weak negative correlation is observed among the scores got in the Coping ability, Dissociation dimension $(\mathrm{r}=-0.24)$ and Experimentation $(\mathrm{r}=-0.24)$. In the end, Machiavellianism/cynicism dimension is significantly positively correlated to Escape $(\mathrm{r}=0.36)$, Dissociation $(\mathrm{r}=0.33)$ and $E_{X}$ perimentation $(\mathrm{r}=0.34)$.

Table 1. Correlational analysis.

\begin{tabular}{|c|c|c|c|c|c|}
\hline CORRELAZIONI & ESCAPISM & DISSOCIATION & IMPACT & EXPERIMENT & ADDICTION \\
\hline AGENCY & -0.14 & -0.24 & 0.15 & -0.07 & -0.10 \\
\hline $\begin{array}{l}\text { SOCIAL/EMOTIONAL } \\
\text { INTELLIGENCE }\end{array}$ & -0.07 & -0.13 & 0.09 & -0.20 & -0.02 \\
\hline SELF-REGULATION & -0.28 & -0.36 & 0.08 & -0.26 & -0.15 \\
\hline COPING ABILITY & -0.19 & -0.24 & -0.09 & -0.24 & -0.13 \\
\hline INNOVATION & -0.09 & -0.07 & 0.05 & -0.15 & -0.10 \\
\hline SELF-ESTEEM & -0.15 & -0.18 & 0.08 & -0.11 & -0.14 \\
\hline SOCIAL DESIRABILITY & -0.16 & -0.13 & -0.11 & -0.14 & -0.19 \\
\hline MACHIAVELLIANISM & 0.36 & 0.33 & -0.15 & 0.34 & 0.29 \\
\hline $\begin{array}{l}\text { IMPRESSION } \\
\text { MANAGEMENT }\end{array}$ & 0.15 & 0.05 & 0.03 & 0.15 & 0.20 \\
\hline
\end{tabular}




\section{Discussion}

The average time spent connected to the Internet is observed to be over 20 hours per week for about $32 \%$ of the subjects in the sample. A minority of them (3\%) state moreover to use Internet for over 40 hours per week. But these data seem to be a sub-evaluation, as the Internet connection is working even if there is not an active use on the individuals' part (e.g. getting/sending messages, emails, and using chat-rooms on the mobile phone). They should not be neglected, because some scholars, among which Young (1998) consider a level of 39 hours per week (not for school and/or job purpose) as a possible cut-off meaning that there is a problem in usage, while other authors suggest an average of 27 hours per week as a clinical cut-off. As for the purposes they use the Net for, the surveyed young people match the statistics that are reported in literature because generally $97 \%$ of them use the Internet mainly to communicate through chat rooms and social networks; 73\% use the Net also for research and to share information. The analysis of the data collected through the UADI test permitted to define the behaviour related to the use of technology by the young people in the sample. For almost the total number of people (97\%), the Net can be used for escaping purposes, but contextually with a psychological condition in which real needs overtake imaginative ones. Otherwise, for $3 \%$ of the people, the use of the Net means escaping from problems, and compensation to the difficulties in everyday life, and becomes a difficulty in establishing social satisfactory relationships. $2 \%$ of the sample seems to present bizarre sensory perceptions, depersonalisation, alienation and escape from reality when they are connected, while almost the total number of the people present no dissociative symptoms connected to the use of the Net. For the greatest part of the subjects in the sample, the Internet has no, or a very low one, impact on life, and real activities integrate virtual ones; for $1 \%$ of the subjects the Net impact life causing social disease, psychophysical weariness and twisting in habits. The last date is exclusively for the male students sub-group, that gets an average higher score (pathological) on the scale of impact on life. In the young people we surveyed, we can observe normal levels of the tendency to experimentation, a natural need to express themselves in new roles or identities on the Internet that becomes a kind of social laboratory for the Self-experimentation. In this case as well, we must consider that $2 \%$ of the subjects show the tendency to behave in a transgressive and aggressive way online, to lie, to feel excited, or bored. Particularly, with respect to the dimension of the addiction, we can state that the greatest number of the subjects in the sample present a correct management of the Net, connecting according to former decisions, and presenting a steady mood when they use the Internet. Only $1 \%$ of the sample can be considered at risk of internet addiction. The use of the Internet by the digital natives in our sample is mainly not a problematical one, and fall within a way of relationship, communication and information. A percentage of the surveyed subjects equal to $9 \%$ of the sample is otherwise "at risk" of a problematical use of the Internet, and they are the subjects whose scores in the UADI 
scales are higher than the cut-off, at least in one of the 5 scales.

The analysis of the answers to Multidimensional Personality Profile questionnaire permitted to outline a psychological profile of the users considered in the research, also diversified according to the gender. Agency: the male subjects of the sample present on average normal abilities to assert their opinions, to set ambitious enough purposes, to know how to lead and motivate others; they show a proper force and readiness in their activities. On the other side, female subjects seem to meet difficulties to speak in front of a group of people or in public, or to little familiar people. They do not show to have ambitious purposes, to seek success or to try to excel on other people. They are inclined to skip tasks that ask to do more than one activity simultaneously, and show difficulties in taking decisions rapidly. Social/Emotional Intelligence: as the male subjects, as the female ones present normal abilities to put themselves in the others' place, and to perceive and express emotions. Self-Regulation: the subjects show normal abilities to make plans, and persistence towards purposes. Female subjects seem to have a proper ability to regulate their mood and their feelings. They show normal abilities of emotional control, even in the presence of difficulties and stress situations. They show a proper peace of mind and the ability to control the states of their worries, tension and discouragements. They show a normal ability to recover after some failure, disappointment, hard time of difficulties. In the male sub-group, there is on average a higher ability to control their mood, feelings and emotions, as in interpersonal relationships, as in decisions and usual activities. Innovation: the subjects present on average a normal interest and curiosity about cultural activities. They are tolerant in respect to differences and show proper qualities of fantasy and imagination. With regards to the Self-Esteem dimension, the surveyed subjects show a positive attitude towards themselves, a substantial confidence in their abilities, and are naturally proud of themselves and their actions. Social Desirability: the subjects present on average a self-image apparently free from attempts to alterate it positively or negatively. Machiavellianism/cynism: on average, the subjects seem to be able to balance their interests with the interests of other people. To reach their purposes, they tend to adapt their behaviour to circumstances, and to make it compatible with the limits imposed by the purposes of other people. About the last surveyed category, Impression management, the subjects show a normal interest in trying to win the favour of others, to be appreciated and to be trusted.

The correlational analysis reveals a slight negative correlation between the Agency dimension, evaluated through the MPP questionnaire, and the Dissociation dimension in the UADI questionnaire. It can be reasonably supposed that there is a possible inverse relationship between the ability to assert one's own opinions, be successful and to accomplish one's purposes (Agency) and the tendency to dissociate and escape reality by means of the new informatic technologies (Dissociation). There is also a slight negative correlation $(\mathrm{r}=-0.20)$ between the Social/Emotional Intelligence dimension and the Experimentation 
dimension. It can be assumed that the more the subjects are able to empathize with others, to express emotions, and to take into account the needs of other people (Socal/Emotional Intelligence), the less they are inclined to carry out aggressive behaviours and mystify their identity (Experimentation). The Self-Regulation dimension is slightly negatively correlated to Escapism $(\mathrm{r}=-0.28)$, significantly negatively correlated to Dissociation $(\mathrm{r}=-0.36)$ and weakly negatively correlated to Experimentation $(\mathrm{r}=-0.26)$. In this case as well, the negative correlation might point out that the more people are able to set purposes, monitor actions, organise and deal with matters with order and method (Self-Regulation), the less they will tend to use Internet to escape from problems and real difficulties (Escapism), the less they will tend to have dissociative experiences connected to the use of Internet (Dissociation), and the less they will use the Net to alter their identity or to get transgressive experiences. There is also a slight negative correlation between the scores got in the Coping Ability dimension and in the Dissociation dimension $(\mathrm{r}=-0.24)$, and Experimentation $(\mathrm{r}=-0.24)$. It can be supposed that the more the subjects are able to regulate their mood and feelings even in stressing and problematical situations, the less they show the tendency to dissociation, and a pathological need to experiment on the Net. These results seem to be consistent with what is reported by some already mentioned authors (Chak \& Leung, 2004; Kim \& Haridakis, 2009) that identify in the inner locus of control a protective factor very significant for a healthy and effective use of the Internet that is so regarded as a resource, and not as an alternative reality (Wallace, 1999; Turkle 1995). The last correlations to consider are positive ones and are related to the relationship between Machiavellianism and Escapism $(\mathrm{r}=$ 0.36), Dissociation $(\mathrm{r}=0.33)$, and Experimentation $(\mathrm{r}=0.34)$. The positive direction of the correlations points to the tendency of the considered dimensions to increase or decrease together. On this basis, it can be assumed, for example, that to a high level of cynicism also correspond a high tendency to use Internet to escape from reality, a high tendency to dissociation and to aggressive/transgressive behaviours on the Net.

\section{Conclusion}

Computers and informatic technologies seem, like the two-faced Janus, to have a basic ambivalence: if a face looks at progress and evolution, simplification and enrichment of the process of information exchange, the other one is turned to areas at risk, not always easily valuable. The data in this study assert the need not to minimize or deny the possible negative effects of a massive use of the Internet, as it is associated-even if in a reduced (but not negligible) number of cases (9\% of the sample surveyed by the UADI questionnaire) - to the risk of psychological problems that can impact effectively on the life of the subjects. Also, a time connection exceeding 20 hours a week characterizes the Internet usage by young people at risk (in this case $32 \%$ ). With increasing the connection time, there is indeed an increasing of the risk of abuse or problematic use of the Internet, but, 
given the pervasiveness of this technological tool, (that is also used to study and to work), it is not easy to define a precise borderline between normality and pathology. But it seems important, anyway, not to "pathologize" the use of internet, as the results show that the greatest number of the subjects can manage technology effectively and can use it properly for their purposes. As concerns the relationship between personality characteristics and the use/abuse of internet we can conclude, on the basis of the data obtained, that there is a possible inverse relationship between the ability to assert one's own opinions, be successful and to accomplish one's purposes (Agency) and the tendency to dissociate and escape reality by means of the new informatic technologies (Dissociation). The data also reveal that the more people are able to set purposes, monitor actions, organise and deal with matters with order and method (Self-Regulation), the less they will tend to use Internet to escape from problems and real difficulties ( $E s$ capism), the less they will tend to have dissociative experiences connected to the use of Internet (Dissociation), and the less they will use the Net to alter their identity or to get transgressive experiences. Finally, a high level of cynicism seems to be associated with a high tendency to use Internet to escape from reality, a high tendency to dissociation and to aggressive/transgressive behaviours on the Net.

\section{References}

Aboujaoude, E., Koran, L. M., Gamel, N., Large, M. D., \& Serpe, R. T. (2006). Potential Markers for Problematic Internet Use: A Telephone Survey of 2513 Adults. CNS Spectr, 11, 750-755. https://doi.org/10.1017/S1092852900014875

Caplan, S. E. (2002). Problematic Internet Use and Psychosocial Well-Being: Development of a Theory-Based Cognitive-Behavioral Measurement Instrument. Computers in Human Behavior, 18, 553-575. https://doi.org/10.1016/S0747-5632(02)00004-3

Caprara, G. V., Barbaranelli, C., De Carlo, N. A., \& Robusto, E. (2006). Multidimensional Personality Profile (MPP). A New Generation Questionnaire for the Assessment of Personality. Milano, Italy: Franco Angeli.

Caretti, V., La Barbera, D., Craparo, G., \& Mangiapane, E. (2005). The Correlation between Alexithymia and Dissociation in Pathological Addiction. New Perspectives of Research on Addiction. In V, Caretti., \& D., La Barbera (Eds.), Pathological Addictions. Clinic and Psychopathology (pp. 249-278). Milano, Italy: Raffaello Cortina Editore.

Chak, K., \& Leung, L. (2004). Shyness and Locus of Control as Predictors of Internet Addiction and Internet Use. Cyberpsychology \& Behavior, 7, 559-570. https://doi.org/10.1089/cpb.2004.7.559

Chou, C., \& Hsiao, M.-C. (2000). Internet Addiction, Usage, Gratification, and Pleasure Experience: The Taiwan College Students' Case. Computers and Education, 35, 65-80. https://doi.org/10.1016/S0360-1315(00)00019-1

Del Miglio, C., Gamba, A., \& Cantelmi, T. (2001). Costruzione e validazione preliminare di uno strumento (U.A.D.I.) per la rilevazione delle variabili psicologiche e psicopatologiche correlate all'uso di internet. Giornale Italiano di Psicopatologia, 7, 293-306.

Durkee, T., Kaess, M., Carli, V., Parzer, P., Wasserman, C., Floderus, B., Apter, A., Balazs, J., Barzilay, S., Bobes, J., Brunner, R., Corcoran, P., Cosman, D., Cotter, P., Despalins, R., Graber, N., Guillemin, F., Haring, C., Kahn, J. P., Mandelli, L., Marusic, D., 
Mészáros, G., Musa, G. J., Postuvan, V., Resch, F., Saiz, P. A., Sisask, M., Varnik, A., Sarchiapone, M., Hoven, C. W., \& Wasserman, D. (2012). Prevalence of Pathological Internet Use among Adolescents in Europe: Demographic and Social Factors. Addiction, 107, 2210-2222. https://doi.org/10.1111/j.1360-0443.2012.03946.x

Eppright, T., Allwood, M., Stern, B., \& Theiss, T. (1999). Internet Addiction: A New Type of Addiction? Missouri Medicine, 96, 133-136.

Fiumana, V., Moschetti, C., Alimonti, E., Corvino, S., Distefano, E., \& Tonioni, F. (2011). Dipendenza da internet e alessitimia: un'ipotesi di associazione e osservazione sperimentale. Dipendenze patologiche, 3, 21-30.

Greenfield, D. N. (1999). Psychological Characteristics of Compulsive Internet Use: A Preliminary Analysis. Cyberpsychology and Behavior, 2, 403-412.

https://doi.org/10.1089/cpb.1999.2.403

Khazaal, Y., Billieux, J., Thorens, G., Khan, R., Louati, Y., Scarlatti, E., Theintz, F., Lederrey, J., Van Der Linden, M., \& Zullino, D. (2008). French Validation of the Internet Addiction Test. Cyberpsychology, Behavior and Social Networking, 11, 703-706. https://doi.org/10.1089/cpb.2007.0249

Kim, J., \& Haridakis, P. M. (2009). The Role of Internet Use Characteristics and Motives in Explaining Three Dimensions in Internet Addiction. Journal of Computer-Mediated Communication, 3, 988-1015. https://doi.org/10.1111/j.1083-6101.2009.01478.x

Ko, C. H., Yen, J. Y., Chen, C. C., Chen, S. H., \& Yen, C. F. (2007). Factors Predictive for Incidence and Remission of Internet Addiction in Young Adolescents: A Prospective Study. Cyberpsychology and Behavior, 10, 545-551.

https://doi.org/10.1089/cpb.2007.9992

Leung, L. (2004). Net-Generation Attributes and Seductive Properties of the Internet as Predictors of Online Activities and Internet Addiction. Cyberpsychology \& Behavior, 7, 333-348. https://doi.org/10.1089/1094931041291303

McKenna, K. Y. A., \& Bargh, J. A. (2000). Plan 9 from Cyberspace: The Implication of the Internet for Personality and Social Psychology. Personality and Social Psychology Review, 4, 57-75. https://doi.org/10.1207/S15327957PSPR0401_6

Morahan-Martin, J., \& Schumacher, P. (2000). Incidence and Correlates of Pathological Internet Use among College Students. Computers in Human Behavior, 16, 13-29. https://doi.org/10.1016/S0747-5632(99)00049-7

Papacharissi, Z., \& Rubin, A. M. (2000). Predictors of Internet Use. Journal of Broadcasting \& Electronic Media, 44, 175-196. https://doi.org/10.1207/s15506878jobem4402_2

Parker, J. D., Wood, L. M., Bond, B. J., \& Shanghnessy, P. (2005). Alexithymia in Young Adulthood: A Risk Factor for Pathological Gambling. Psychotherapy Psychosomatic, 74, 51-55. https://doi.org/10.1159/000082027

Perrella, R., \& Caviglia, G. (2014). Internet Addiction. Adolescent and Adults. Santarcangelo di Romagna: Maggioli Editore.

Poli, R., \& Agrimi, E. (2012). Internet Addiction Disorder: Prevalence in an Italian Students Population. Nordic Journal of Psychiatry, 66, 55-59.

https://doi.org/10.3109/08039488.2011.605169

Rothbart, M. K., \& Bates, J. E. (2006). Temperament. In W. Damon, R. M. Lerner, N. Eisenberg (Eds.), Handbook of Child Psychology (6th ed., pp. 99-166). New York, NY: Wiley.

Tonioni, F., D’Alessandris, L., Lai, C., Martinelli, D., Corvino, S., Vasale, M., Fanella, F., Aceto, P., \& Bria, P. (2011). Internet Addiction: Hours Spent Online, Behaviors and Psychological Symptoms. General Hospital Psychiatry, 34, 80-87.

https://doi.org/10.1016/j.genhosppsych.2011.09.013 
Tsai, C. C., \& Lin, S. S. (2003). Internet Addiction of Adolescents in Taiwan: An Interview Study. Cyberpsychology and Behavior, 6, 649-652.

https://doi.org/10.1089/109493103322725432

Turkle, S. (1995). Life on the Screen. New York, NY: Simon \& Schuster.

Velezmoro, R., Lacefield, K., \& Roberti, J. W. (2010). Perceived Stress, Sensation Seeking and College Students' Abuse of the Internet. Computers in Human Behavior, 26, 1526-1630. https://doi.org/10.1016/j.chb.2010.05.020

Wallace, P. (1999). The Psychology of the Internet. New York, NY: Cambridge University Press. https://doi.org/10.1017/CBO9780511581670

Widyanto, L., \& McMurran, M. (2004). The Psychometric Properties of the Internet Addiction Test. CyberPsychology \& Behavior, 7, 443-450. https://doi.org/10.1089/cpb.2004.7.443

Yen, J., Yen, C., Chen, C., Chen, S., \& Ko, C. (2007). Family Factors of Internet Addiction and Substance Use Experience in Taiwanese Adolescents. Cyberpsychology \& Behavior, 10, 323-329. https://doi.org/10.1089/cpb.2006.9948

Young, K. S. (1998). Caught in the Net. Chichester: Wiley. 